Dhaka Univ. J. Sci. 61(1): 117-123, 2013 (January)

\title{
Effect of Heat Treatment on Dilute Solution Properties of Sugar Beet Pectin Kazi S.Rahman ${ }^{1 \oplus}$, Khandker S. Hossain ${ }^{1 *}$ A. Takada ${ }^{2}$, Y. Takahashi ${ }^{2}$ and K. Nishinari ${ }^{3}$ \\ ${ }^{1}$ Department of Physics, University of Dhaka, Dhaka-1000, Bangladesh. \\ ${ }^{2}$ Institute for Materials Chemistry and Engineering, Kyushu University, Fukuoka 816-8580, Japan \\ ${ }^{3}$ Graduate School of Human Life Science, Osaka City University, Osaka, Japan
}

Received on 20.04.2012. Accepted for Publication on 24.05.2012

\begin{abstract}
We investigate the conformational changes of pectin molecules in aqueous solution due to heat treatment of the powdered samples of Sugar Beet Pectin (SBP) polysaccharide. Aqueous solution properties of these samples have been studied using Ubbelohde capillary viscometer in the dilute regime. Intrinsic viscosity $[\eta]$ was estimated for different SBP solution samples prepared from heat-treated SBP powdered samples with different heating time. Results suggest that heat treatment has a significant effect on solution behavior of SBP samples. Intrinsic viscosities decreased with increasing heating time indicating that heat-treated SBP molecules are compact than the untreated one and the compactness increases with increasing heating time at $80^{\circ} \mathrm{C}$. The intrinsic viscosities of these samples were plotted as a function of the corresponding molecular weight of the samples and can be expressed by a power law. The power law exponent was estimated as 1.4 indicating that pectin molecules takes the conformation of stiff polymer chains in dilute solution.
\end{abstract}

\section{Introduction}

Pectin is a natural polysaccharide obtained from citrus peel, apple pomace or sugar beet pulp as a byproduct during the extraction of sugar. ${ }^{1}$ The major constituent of pectin is a linear sequence of 1,4-linked $\alpha$-D-galacturonyl units (usually referred to as "smooth regions") occasionally interrupted by 1,2-linked $\alpha$ - L -rhamnopyranosyl residues and with some of the carboxyl groups esterified with methanol. The chains also have branches (referred to as "hairy regions") in minor proportions consisting of neutral sugars, particularly galactose and arabinose. ${ }^{2-4}$ Commercial pectins are obtained by acid extraction and used as thickener, gelling agent, stabilizer, and emulsifier in the food industry and pharmaceutical applications. ${ }^{5-7}$

Sugar beet pectin (SBP) ${ }^{8-9}$ differs from other pectins in that it has a higher proportion of neutral sugar side chains, acetic acid content, phenolic esters and a higher content of protein material bound to the side chains through covalent linkages. ${ }^{10-11}$ The gel forming ability of SBP is very poor in contrast to other types of pectin obtained from different sources. The factors that may affect gelation in SBP are amount of sugars, proteins, ferulic acid, degree of esterification, degree of acetylation, degree of blockiness, $\mathrm{pH}$, amount of $\mathrm{Ca}^{2+}$ ions, and Molecular weight, etc.

However, SBP is reported to show a considerable emulsification ability due to its more hydrophobic nature where the proteinaceous materials in the pectin molecules activated the oil-water interface. ${ }^{12}$ The presence of higher content of protein material in SBP allow us to explore the widespread application by heat-treating the sample at temperature higher than $80^{\circ} \mathrm{C}$ since a heat induced denaturation usually occurs in proteins at $T$ higher than $80^{\circ} \mathrm{C}^{13}$ In this connection, it is not unexpected to assume that an effect of the heat treatment on the dilute solution properties such as size of the single molecule and cluster, interaction of the molecule with solvent etc may exists.

The physical properties of a polymer solution depend on solvent, temperature, and concentration. At low concentrations, the polymer chains are separated from each other, where each chain occupies a spherical volume of radius $R_{\mathrm{g}}$. The hydrodynamic volume occupied by a given polymer mass is the intrinsic viscosity, $[\eta]$, which is a parameter that can be determined by dilute solution viscosity measurements. Intrinsic viscosity probes the interaction of molecular structure with the solution. Several theories of polymer physics ${ }^{14-15}$ relate intrinsic viscosity to molecular properties of polymers such as molecular weight, overlap concentration, radius of gyration, and pore size of concentrated polymers. The intrinsic viscosity values are important for probing biological macromolecular structure and interaction with solution and to characterize the chain shape or the conformational transition of polymer molecules in solution ${ }^{16-17}$

A considerable amount of studies has been done in order to elucidate the solution properties of pectin including SBP using intrinsic viscosity behavior. ${ }^{18-20}$ However, to the best of our knowledge, such study on the heat-treated SBP is still an early stage. The conformational changes in SBP can be caused by heat treatment which is expected to show a change in viscosity behavior or rheological behavior of SBP solution. The present investigation is aimed at to characterization of the heat treated SBP samples in aqueous solution with different concentrations using Ubbelohde capillary viscometer at a constant temperature of $T=25^{\circ} \mathrm{C}$.

\section{Experimental Section}

\section{Materials and sample preparation}

Unmodified and modified (Heat-treated) Sugar beet pectin samples were kindly supplied by San-eigen F.F.I Inc. Japan

\footnotetext{
* To whom corresponding should be addressed; e-mail: k.s.hossain@gmail.com

${ }^{\oplus}$ Present Address: Jagannath University, Dhaka-1100, Bangladesh
} 
and were used in this study without further purification. The molecular weight distribution was determined by SECMALLS. Here we used one unmodified sample and four modified samples by heat-treatment. The unmodified and modified samples will be referred to hereafter as SBP and (SBP-A, SBP-B, SBP-C, SBP-D), respectively. Unmodified SBP were heat-treated at $80^{\circ} \mathrm{C}$ for different heating conditions as shown in Table 1. Some characterizing parameters of the samples are given in Table 2 as supplied by the manufacturer. For getting best result distilled water are used here for preparing solution.

\section{Table. 1. Different Sugar Beet Pectin (SBP) Samples}

\begin{tabular}{|c|c|c|c|}
\hline $\begin{array}{c}\text { Sample } \\
\text { Code }\end{array}$ & $\begin{array}{c}\text { Heating } \\
\text { Time } \\
\text { In Hours }\end{array}$ & $\begin{array}{c}\text { Treated at } \\
\text { temperature } \\
\left({ }^{\circ} \mathbf{C}\right)\end{array}$ & $\begin{array}{c}\text { Humidity } \\
(\%)\end{array}$ \\
\hline SBP & Unmodified & Unmodified & Unmodified \\
\hline SBP-A & 5 & 80 & 70 \\
\hline SBP-B & 8 & 80 & 70 \\
\hline SBP-C & 16 & 80 & 70 \\
\hline SBP-D & 24 & 80 & 70 \\
\hline
\end{tabular}

Table . 2. Characterization of the samples by SEC MALLS (Supplied by the manufacturer of the sample)

\begin{tabular}{|c|c|c|c|c|c|}
\hline Sample & $\begin{array}{c}\text { Heating } \\
\text { Time } \\
/ \text { Hour }\end{array}$ & $\begin{array}{c}\text { Aqueous } \\
\text { viscosity } \\
/ \mathbf{m P a} \text { a.s }\end{array}$ & $\mathbf{p H}$ & $\begin{array}{c}\boldsymbol{M}_{\mathbf{w}} \\
/ \mathbf{K g} / \mathbf{m o l}\end{array}$ & $\begin{array}{c}\boldsymbol{R}_{\mathbf{g}} \\
\mathbf{/ n m}\end{array}$ \\
\hline Unmodified & 0 & 61.6 & 3.39 & 491.7 & 37.9 \\
\hline SBP-A & 5 & 85.8 & 3.38 & 513.2 & 40.2 \\
\hline SBP-B & 8 & 100 & 3.35 & 493.1 & 40.9 \\
\hline SBP-C & 16 & 197 & 3.35 & 383.9 & 43.6 \\
\hline SBP-D & 24 & 175.5 & 3.33 & 271.2 & 39.3 \\
\hline
\end{tabular}

The powdered pectin samples were dissolved in distilled water and aqueous solution of pectin with the desired concentration was prepared by stirring with a magnetic stirrer at temperature of $25^{\circ} \mathrm{C}$ for about 2 hours to ensure complete dissolution. First of all, a $0.1 \mathrm{wt} \%$ solution of sugar beet pectin was prepared and the solution was mixed with the same amount of water to make $C=0.05 \mathrm{wt} \%$ sample. In this way, the solution samples were diluted with distilled water to make rest of the samples. A total of 4 concentration samples with $C=0.0125 \mathrm{wt} \%, 0.025 \mathrm{wt} \%, 0.05 \mathrm{wt} \%$ and $0.1 \mathrm{wt} \%$ were prepared at the room temperature of $25^{\circ} \mathrm{C}$ for unmodified and modified SBP samples.

\section{Method}

Solutions of a high-molecular-weight polymer, even at low concentrations, can flow only slowly. Addition of a small amount of the polymer to the fluid can make it viscous, thereby preventing unwanted turbulence in the flow. The proportionality coefficient $\eta$ is called the viscosity. Viscosity depends not only on the molecular mass but also on concentration and temperature.

The capillary viscometric measurements were performed using an Ubbelohde viscometer at the constant temperature of $(25 \pm 0.5){ }^{\circ} \mathrm{C}$. The capillary viscometer was filled with 5 $\mathrm{ml}$ of sample and equilibrated in a water bath which was under precise temperature control with a temperature controller (CHINO) to maintain temperatures at $25^{\circ} \mathrm{C}$. The sample was passed through the capillary once before the running time was measured. The running times of each solution and solvent were measured 5 times and were recorded with an accuracy of $\pm 0.05 \mathrm{~s}$. The time it took for a volume of polymer solution to flow through a thin capillary was compared to the time for a solvent flow. It turns out that the flow time for either is proportional to the viscosity, and inversely proportional to the density.

$t_{s}=\frac{\eta_{s}}{\rho_{s}}$

$t=\frac{\eta}{\rho}$

where the parameters with subscript $\mathrm{s}$ represents solvent and without subscripts represents solution.

When the concentration $C$, expressed in $\mathrm{g} / \mathrm{L}$, is sufficiently low, the viscosity of the solution is not much different from the viscosity of the pure solvent.

We define the relative viscosity denoted by $\eta_{\mathrm{r}}$ to be the ratio $\eta / \eta_{s}$. For most polymer solutions at the concentrations of interest, $\rho / \rho_{s} \approx 1$. Thus, to a very good approximation, the relative viscosity is a simple time ratio:

$$
\eta_{r}=t / t_{s}
$$

It is to be noted here that although the ratio of solution viscosity to solvent viscosity is dimensionless, it is customary to use the symbol $\eta_{\mathrm{r}}$ for the relative viscosity.

We also define a "specific viscosity", $\eta_{\text {sp }}$, to be the fractional change in viscosity upon addition of polymer given by the following equation.

$$
\eta_{\mathrm{sp}}=\eta_{\mathrm{r}}-1
$$

where $\eta_{\mathrm{sp}}=\left(\eta-\eta_{\mathrm{s}}\right) / \eta_{\mathrm{s}}$

We also define reduced viscosity, $\eta_{\text {red, }}$ and inherent viscosity, $\eta_{\text {in }}$, by $\eta_{\text {sp }} / C$ and $\left(\ln \eta_{\mathrm{r}}\right) / C$, respectively,

The Ubbelohde capillary viscometer technique allows us to calculate the specific viscosity and relative viscosity from the data obtained for different SBP samples with various polymer concentrations of solutions ${ }^{14}$. 


\section{Results and Discussion}

\section{Viscosity behavior of SBP aqueous solution samples}

The viscosity measurement is one of the most widely used approaches for the characterization of both synthetic and biological macromolecules in solution. Each macromolecule in a solution contributes to viscosity. The solution viscosity, $\eta$, increases above the solvent viscosity, $\eta_{\mathrm{s}}$, linearly with polymer concentration, $C$, in extreme dilute solutions where the contribution is additive. Hence, the solution viscosity can be described by the Huggins ${ }^{17}$ and Kraemer equations: ${ }^{21}$

$$
\begin{aligned}
& \frac{\eta-\eta_{\mathrm{s}}}{\eta_{s} C}=\frac{\eta_{s p}}{C}=\eta_{\text {red }}=[\eta]+K_{H}[\eta]^{2} C \\
& \frac{\ln \left(\eta / \eta_{s}\right)}{C}=\frac{\ln \eta_{r}}{C} \eta_{i n}=[\eta]+K_{K}[\eta]^{2} C
\end{aligned}
$$

where $K_{\mathrm{H}}$ and $K_{\mathrm{K}}$ are the Huggins and the Kraemer coefficients, respectively. The intrinsic viscosity, $[\eta]$, which represents the hydrodynamic volume occupied by a given polymer mass, is defined as the limiting value of either the reduced viscosity, $\eta_{\text {red }}$, or inherent viscosity, $\eta_{\text {in }}$, as both the shear rate and polymer concentration, $C$, approach zero which is intended to reflect the influence of the polymer on the solvent when polymer-polymer interactions become progressively less significant. ${ }^{22}$ Thus the intrinsic viscosity of polymer can be expressed mathematically as

$$
[\eta]=\lim _{C \rightarrow 0}\left(\eta_{\text {red }}\right)=\lim _{C \rightarrow 0}\left(\eta_{\text {in }}\right)
$$

The intrinsic viscosity depends on molecular weight and structure of the dissolved polymer, ionic strength and hydrophobicity of solvent, and temperature of the system. ${ }^{15}$

The $\eta_{\mathrm{sp}}$ and $\eta_{\mathrm{r}}$ clearly depend on the concentration of the SBP in solutions. Measuring at zero concentration $(C=0)$ would be useless, but this concept of extrapolating to $C=0$ is very important in polymer characterization and in thermodynamics generally.

Fig. 1 shows the plot of reduced viscosity, $\eta_{\text {red, }}$ and inherent viscosity, $\eta_{\text {in }}$, as a function of concentration for the unmodified SBP sample at a fixed temperature of $25^{\circ} \mathrm{C}$. From the $\eta_{\text {red }}$ vs $C$ data the SBP solutions showed a decreasing tendency with dilution of SBP, and this behavior can be attributed to the contraction of the molecules with dilution indicating an absence of charge-charge repulsions along the chain. The contraction of the molecules with dilution can also be attributed to the polymer solvent interaction. The positive slope appeared in the $\eta_{\text {red }}$ vs $C$ data also indicates that distilled water used in this study act as poor solvent for SBP samples. In a poor solvent segments of a polymer molecule attract each other in solution more strongly than attract the sorrounding solvent molecules. The polymer molecule assumes a tighter configuration and the solution has a lower intrinsic viscosity.

As shown in Fig. 1 , the $\eta_{\text {in }}=1 / C \ln \eta_{\text {rel }}$ shows a decreasing tendency with decreasing concentration with weaker positive slope. The $\eta_{\mathrm{sp}} / C \& 1 / C \ln \eta_{\mathrm{rel}}$ vs. $C$ plot is a linear plot usually obtained for neutral polymer solution. After extrapolating to zero concentration, the both plots should have the same intercept, which is the intrinsic viscosity, $[\eta]$ of this unmodified pectin's solution. In this study, such an attempt was made, however, the two plots did not have the same intercept. Therefore linear regression extrapolations to zero polymer concentration were carried out using reduced viscosity and inherent viscosity data. Table 3 shows the intrinsic viscosity values obtained by the two methods, together with the calculated average, thus providing a more representative derivation for intrinsic viscosity. The intrinsic viscosity, $[\eta]$ of this unmodified pectin's solution was estimated as $398 \mathrm{~mL} / \mathrm{g}$ which suggests the volume occupied by $1 \mathrm{gm}$ of unmodified SBP is $398 \mathrm{~mL}$.

Using the same procedure the dependence of $\eta_{\mathrm{sp}} / C \&$ $\left(1 / C \ln \eta_{\text {rel }}\right)$ as a function of concentration, $C$, are plotted in Fig. 2 for four modified samples by heat treatment. Similar trends i.e. a positive slope in the $C$ dependence of $\eta_{\mathrm{sp}} / C \&$ $\left(1 / C \ln \eta_{\text {rel }}\right)$ for all the pectin samples was observed although there is a difference in slope values and the intercept of each curve corresponding to the different samples. Since the intercepts of the two plots did not coincide also for modified samples, we followed the similar methods for determining the intrinsic viscosity $[\eta]$ values of the other modified SBP solutions prepared by heat treatment. The values are listed up in Table 3.

The values of $[\eta]$ of both unmodified and modified SBP samples are shown in Fig. 3 where the $[\eta]$ of modified SBP samples are plotted against the heating time.

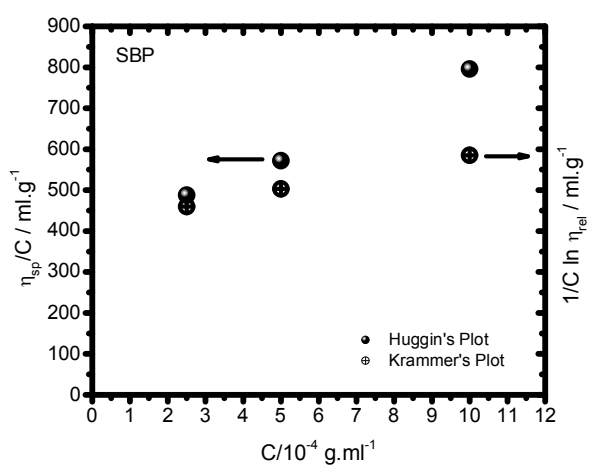

Fig. 1. Plot of the reduced viscosity $\eta_{\mathrm{red}}=\eta_{\mathrm{sp}} / c$ and inherent viscosity $\eta_{\text {in }}=\ln \eta_{\text {rel }} / c$ vs concentration for the Unmodified Sugar Beet Pectin (SBP) aqueous solution. Extrapolation to zero concentration is done using linear regression analysis. 

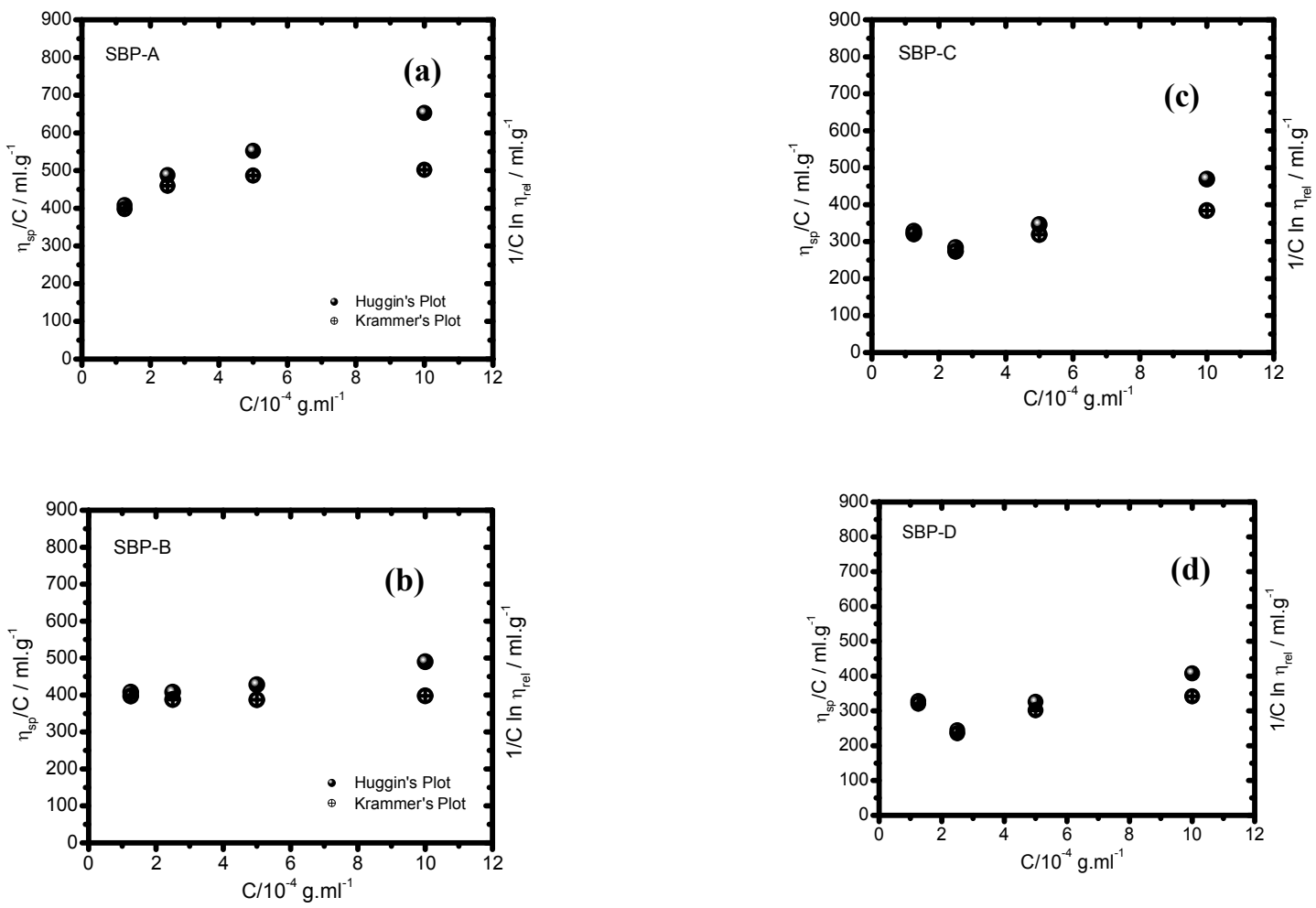

Fig. 2. Plot of the reduced viscosity $\eta_{\mathrm{red}}=\eta_{\mathrm{sp}} / c$ and inherent viscosity $\eta_{\mathrm{in}}=\ln \eta_{\mathrm{rel}} / c$ vs concentration of the Heat-treated modified Pectin aqueous solutions shown in (a) SBP-A, (b) SBP-B, (c) SBP-C, and (d) SBP-D. Extrapolation to zero concentration is done using linear regression analysis.

Table. 3. Different viscosity parameters from Sugar Beet Pectin (SBP) Samples

\begin{tabular}{|c|c|c|c|c|c|c|c|c|c|c|}
\hline Sample & $\begin{array}{c}\mathrm{C} \\
\text { /g.mL } \\
\mathbf{x} 10^{-4}\end{array}$ & $\begin{array}{c}\text { Time } \\
\text { for } \\
\text { solution } \\
t_{\mathrm{s}} / \mathrm{s}\end{array}$ & $\begin{array}{c}\text { Time } \\
\text { for } \\
\text { water } \\
t_{\mathrm{w}} / \mathrm{s}\end{array}$ & $\begin{aligned} & \eta_{\mathrm{r}} \\
= & t_{\mathrm{s}} / t_{\mathrm{w}}\end{aligned}$ & $\begin{array}{l}\eta_{\mathrm{sp}}= \\
\eta_{\mathrm{r}}-1\end{array}$ & $\begin{array}{c}\eta_{\mathrm{sp}} / \mathrm{C} \\
(\mathrm{mL} / \mathrm{g})\end{array}$ & 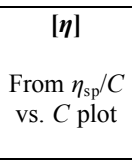 & $\begin{array}{c}1 / C \ln \eta_{\mathrm{r}} \\
(\mathrm{mL} / \mathrm{g})\end{array}$ & $\begin{array}{c}{[\boldsymbol{\eta}]} \\
\\
\text { From } \\
1 / C \ln \eta_{\mathrm{r}} \\
\text { vs. } C \text { plot } \\
\end{array}$ & $\begin{array}{c}\text { Average } \\
{[\eta]}\end{array}$ \\
\hline \multirow{3}{*}{ SBP } & 2.50 & 110 & \multirow{3}{*}{98} & 1.122 & 0.122 & 488 & \multirow{3}{*}{376} & 460 & \multirow{3}{*}{420} & \multirow{3}{*}{398} \\
\hline & 5.00 & 126 & & 1.286 & 0.286 & 572 & & 503 & & \\
\hline & 10.0 & 176 & & 1.796 & 0.796 & 796 & & 585 & & \\
\hline \multirow{4}{*}{ SBP-A } & 1.25 & 103 & \multirow{4}{*}{98} & 1.051 & 0.051 & 408 & \multirow{4}{*}{403} & 398 & \multirow{4}{*}{415} & \multirow{4}{*}{409} \\
\hline & 2.50 & 110 & & 1.122 & 0.122 & 488 & & 460 & & \\
\hline & 5.00 & 125 & & 1.276 & 0.276 & 552 & & 487 & & \\
\hline & 10.0 & 162 & & 1.653 & 0.653 & 653 & & 502 & & \\
\hline \multirow{4}{*}{ SBP-B } & 1.25 & 103 & \multirow{4}{*}{98} & 1.051 & 0.051 & 408 & \multirow{4}{*}{388} & 397 & \multirow{4}{*}{390} & \multirow{4}{*}{389} \\
\hline & 2.50 & 108 & & 1.102 & 0.102 & 408 & & 388 & & \\
\hline & 5.00 & 119 & & 1.214 & 0.214 & 428 & & 387 & & \\
\hline & 10.0 & 146 & & 1.490 & 0.490 & 490 & & 398 & & \\
\hline \multirow{4}{*}{ SBP-C } & 1.25 & 102 & \multirow{4}{*}{98} & 1.041 & 0.041 & 328 & \multirow{4}{*}{267} & 321 & \multirow{4}{*}{279} & \multirow{4}{*}{273} \\
\hline & 2.50 & 105 & & 1.071 & 0.071 & 284 & & 274 & & \\
\hline & 5.00 & 115 & & 1.173 & 0.173 & 346 & & 319 & & \\
\hline & 10.0 & 144 & & 1.469 & 0.469 & 469 & & 384 & & \\
\hline \multirow{4}{*}{ SBP-D } & 1.25 & 102 & \multirow{4}{*}{98} & 1.041 & 0.041 & 328 & \multirow{4}{*}{263} & 321 & \multirow{4}{*}{269} & \multirow{4}{*}{266} \\
\hline & 2.50 & 104 & & 1.061 & 0.061 & 244 & & 236 & & \\
\hline & 5.00 & 114 & & 1.163 & 0.163 & 326 & & 302 & & \\
\hline & 10.00 & 138 & & 1.408 & 0.408 & 408 & & 342 & & \\
\hline
\end{tabular}




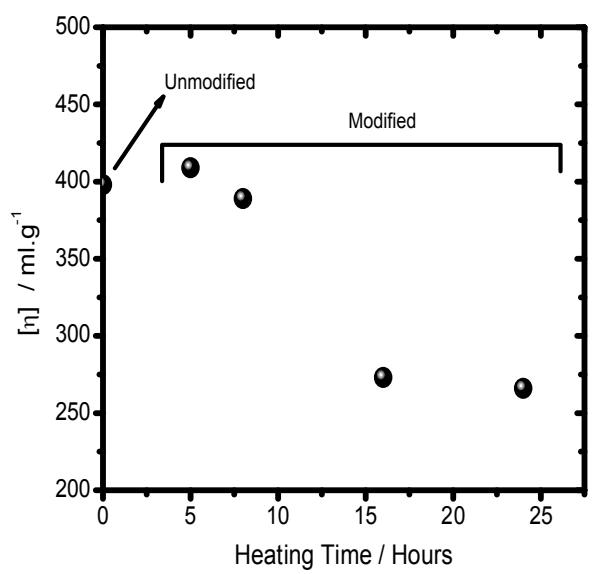

Fig. 3. Dependence of intrinsic viscosity of modified SBP samples by heat treatment is plotted against heating time of the powder sample at $80^{\circ} \mathrm{C}$. The $[\eta]$ of unmodified sample is also shown for comparison.

Figure shows that SBP-A sample with 5 hours of heattreatment time has a $[\eta]$ value of $409 \mathrm{~mL}^{-1}{ }^{-1}$. As heating time increases, $[\eta]$ monotonically decreases with heating time up to 16 hours and then becomes level off and takes the $[\eta]$ value $266 \mathrm{~mL} \cdot \mathrm{g}^{-1}$ for the SBP-D samples with the heating time of 24 hours. In our opinion, the maximum denaturation of SBP occurs near the heating time of 6 hours. Therefore, no further effect of heating time is observed even at 24 hours of heating. The $[\eta]$ values determined in this study are in general agreement with previous studies on citrus pectins. $^{23}$

Although $[\eta]$ is considered to be a measure of molecular weight, it is in fact a measure of the hydrodynamic volume occupied by a molecule. Therefore the decrease in intrinsic viscosity $[\eta]$, indicating a decrease in hydrodynamic volume occupied by a molecule or molecular aggregates composed of these molecules. This tendency can be explained as the following manner.

Sugar beet pectin chain backbone contains a higher content of proteinaceous materials bound to the side chains through covalent linkages. Since the intrinsic viscosity provides information on the overall size and shape of the molecule, it is a sensitive and simple indicator of denaturation. It is likely that heat treatment may cause the side chain of these samples at first to expand and then denature the proteinaceous material in SBP and therefore the pectin molecules in solution may take compact structure which in turn may decrease the polymer's viscosity-enhancing effect in solution.

The compactness of the heat treated SBP molecules may also due to side by side aggregation of Sugar beet pectin molecules due to hydrophobic interaction. At present we are investigating Scanning Electron Microscopy studies in order to clarify the association properties which will be reported soon. The decrease in intrinsic viscosity of biopolymers with other function such as with increasing ionic strength was attributed to conformational changes within the polymer, which could result in a more compact molecule. ${ }^{24}$

\section{Molecular weight dependence of intrinsic viscosity}

Molecular weight dependence of intrinsic viscosity generally obeys power law behavior which allows us to characterize polymer molecules in solution. The scaling relation between intrinsic viscosity $[\eta]$ and molecular weight $M$ widely known as Mark-Houwink-Sakurada equation can be given by the following equation. ${ }^{15,25}$

$$
[\eta]=K M^{a}
$$

where $K$ and $a$ are both constants. The Mark-HouwinkSakurada equation is applicable to many polymers and extensively used to determine molecular weight. The constants $K$ and $a$ both vary with polymers and solvents. The calculation of Mark-Houwink paramenters is carried out by the graphic representation of the following equation.

$$
\ln [\eta]=\ln K+a \ln M
$$

Thus, log-log plots of $[\eta]$ against molecular weight have the intercept $\log (K)$ and slope $a$. The slope contains information about the shape of the molecules: From this equation the molecular weight of a polymer can be determined from data on the intrinsic viscosity and vice versa.

In our study the intrinsic viscosities of Sugar beet pectin samples were plotted as a function of the corresponding molecular weight of the samples. The molecular weight of the samples was supplied by the manufacturer which is measured using SEC-MALLS as shown in Table 2. Fig. 4 shows such a plot where the intrinsic viscosity increases with the molecular weight as expected and the dependence of molecular weight and the intrinsic viscosity can be expressed by a power law. The power law exponent $a$ was estimated as 1.4.

The Mark-Houwink-Sakurada power law exponent indicates a three dimensional configuration of a polymer chain in the solvent environment. The values of $a$ depend on the particular polymer-solvent system. For most flexible polymers, $0.5 \leq a \leq 0$.8. For semi-flexible polymers, $a \geq 0.8$. For polymers with an absolute rigid rod, such as Tobacco mosaic virus, $a=2.0 .^{26}$

Our value of the exponent $a=1.4$ falls in between the two extreme values corresponding to flexible and rod-like polymer conformation suggests that modified SBP molecules take anisotropic shape and behave like a stiff macromolecules. Morris et al. also observed the power law exponent of 0.61 for sugar beet pectin which they attributed 
it to a semi-flexible conformation. ${ }^{27}$ It is to be noted here that sugar beet pectin samples they used were without any modification by heat treatment. Taking into consideration of their results it can be suggested by our study that the modification by heat treatment promotes a conformational change of SBP from semi-flexible to stiff macromolecules.

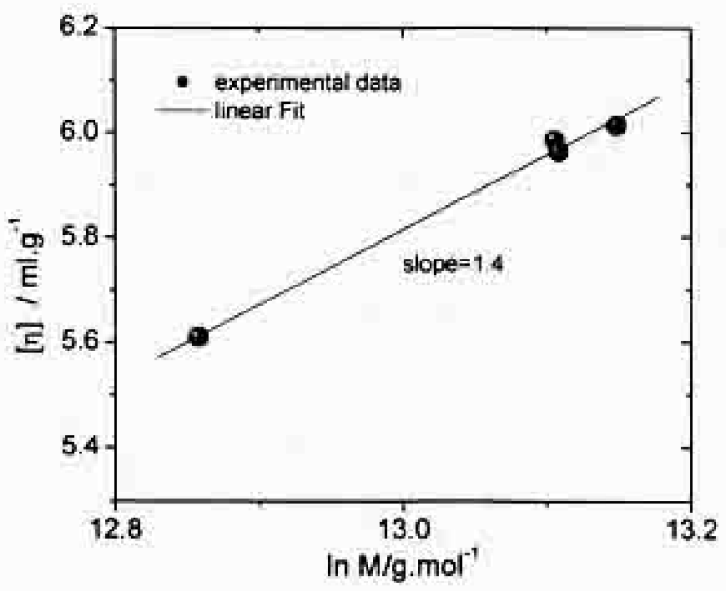

Fig. 4. A plot of Intrinsic viscosity vs molecular weight for SBP samples. The solid line shows the Mark-Houwink-Sakurada equation, where the exponent $a$ is 1.4.

\section{Estimation of overlap concentration of pectin molecules}

The plot of reduced viscosity vs concentration of pectin may define two regions, i.e., dilute and semi-dilute, from an abrupt change in the slope of a straight line at critical concentration $C^{*}$. The $C^{*}$ is also termed as overlap concentration and is a measure of molecular size and conformation of a polymer. The higher the molecular weight and the more rigid is the conformation and lower is the value of $C^{*}$. However such an inflection point was not seen in Fig. 1 and Fig. 2 which indicates that the experiment was conducted in the dilute regime. The inflection point can be observed if we consider some more points in the higher concentration end.

Overlap concentration of polymer chains can also be estimated from the reciprocal of intrinsic viscosity since the intrinsic viscosity is proportional to the reciprocal concentration of monomers in a volume of a polymer chain. Hence, the coil size or radius of gyration of the polymer in a dilute solution can be evaluated using ${ }^{26}$

$$
[\eta] \approx 6.2\left(\frac{R_{g}{ }^{3} N_{A}}{M_{W}}\right)
$$

where $M_{\mathrm{w}}$ is the average molecular weight of polymer chains. As the concentration increases, the polymer coils come closer and start to overlap each other. Since the number of polymer chains per unit volume is $C N_{\mathrm{A}} / M_{\mathrm{w}}$, the concentration, $C^{*}$, at which the overlap starts is estimated as

$$
C^{*}=\frac{3 M_{W}}{4 \pi N_{A} R_{g}^{3}} \approx \frac{1.5}{\lfloor\eta]}
$$

Although $C^{*}$, estimated in eq. 11 , is an adequate criterion for the onset of molecular interactions when the macromolecules are only slightly extended by flow, highly deformed molecules might entangle or otherwise interact at concentrations much lower than this.

A rough estimate of the overlap concentration of all the SBP samples using the eq. 11 has been done and listed up in the Table 4.

Table. 4. Overlap concentration for different Sugar Beet Pectin (SBP) Samples

\begin{tabular}{|c|c|c|}
\hline Sample & $\begin{array}{c}\text { Heating Time } \\
\text { In Hours }\end{array}$ & $\begin{array}{c}\boldsymbol{C}^{*} \\
/ \mathbf{g , m \mathbf { L } ^ { - 1 }}\end{array}$ \\
\hline SBP & Unmodified & $3.77 \times 10^{-3}$ \\
\hline SBP-A & 5 & $3.67 \times 10^{-3}$ \\
\hline SBP-B & 8 & $3.86 \times 10^{-3}$ \\
\hline SBP-C & 16 & $5.50 \times 10^{-3}$ \\
\hline SBP-D & 24 & $5.64 \times 10^{-3}$ \\
\hline
\end{tabular}

Since our experimental concentration range is between $C=1.25 \times 10^{-4} \mathrm{~g} / \mathrm{mL}$ to $C=1.00 \times 10^{-3} \mathrm{~g} / \mathrm{mL}$, it is clear from Table 4 that our experiment was conducted within the dilute solution regime, i.e. we studied the behavior of individual molecule or aggregates made up of these SBP chains which is in consistent with the absence of any inflection point in the reduced viscosity vs concentration curve of Fig. 1 and Fig. 2.

\section{Conclusion}

We carried out measurements of intrinsic viscosity for unmodified and modified SBP as well as comparing older studies. The data obtained clearly shows the effect of heat treatment on dilute solution properties of SBP. Several conclusions are drawn here: (a) Heat-treated SBP molecules are compact than the untreated one and the compactness increases with increasing heating time. Heat treatment may induce side by side aggregation of Sugar beet pectin molecules due to hydrophobic interaction.(b) Modified SBP molecules take anisotropic shape and behave like a stiff macromolecules. (c) The values of $C^{*}$ of SBP confirms that our experiment has been conducted within dilute regime and at concentrations exceeding about $C^{*}$ polymer molecules will touch and interpenetrate. 
These new information may provide insights as to better understand the modification of SBP macromolecule and use it in a wide variety of application in the field of bio-material science, food and pharmaceuticals.

\section{Acknowledgements}

We thank Dr. T. Funami of San- Ei-Gen FFI for providing us with the unmodified and modified SBP samples.

1. Phillips, G. O., and P. A. Williams, 2000. Introduction to food hydrocolloids. :In Handbook of Hydrocolloids, Woodhead Publishing: Cambridge, U.K., p 1.

2. Voragen, A. G. J., W. Pilnik, J. F. Thibault, M. A. V. Axelos, C. M. G. C. Renard, 1995. Pectins. In: A. M. (ed). Food polysaccharides and their applications, Marcel Dekker, New York, 287-339.

3. Oakenfull, D. G., 1991. The chemistry of high-methoxyl pectins : In Walter, R. H.(ed). The Chemistry and Technology of Pectin, Academic Press: New York, 88-108.

4. Morris, V. J. 1986. In Functional Properties of Food Macromolecules; Mitchell, J. R., Ledward, D. A., (ed.). Elsevier Applied Science Publishers: London, Chapter 3, p 121.

5. May, C. D., 2000. Pectin. In G. O. Phillips, P. A. Williams, (ed.). Handbook of Hydrocolloids, Woodhead Publishing, Cambridge, U.K. p 169

6. Ashford, M., J. Fell, D. Attwood, H. Sharma, P. Woodhead, 1994. Studies on pectin formulations for colonic drug delivery. J. Controlled Release, 30, 225.

7. Rubinstein, A., R. Radai, M. Ezra, S. Pathak, J.S. Rokem, 1993. In vitro evaluation of calcium pectinate: a potential colon-specific drug delivery carrier Pharm. Res., 10, 258-263.

8. Ralet, M. C., J. F. Thibault, C.B. Faulds and G. Williamson., 1994. Carbohydr. Res., 263, 227-241.

9. Renard, Catherine M.G.C and Jean-François Thibault, 1993. Structure and properties of apple and sugar-beet pectins extracted by chelating agents. Carbohydrate Research, 244, 99-114

10. Colquhoun, Ian J., Marie-Christine Ralet, JeanFrançois Thibault, Craig B. Faulds, Gary Williamson, 1994. Structure identification of feruloylated oligosaccharides from sugar-beet pulp by NMR spectroscopy. Carbohydrate Research, 263, 243-256.

11. Sakamoto, T. and T. Sakai, 1995. Analysis of structure of sugar-beet pectin by enzymatic methods. Phytochemistry, 39, 821-823.
12. Funami, T., G. Zhang, M. Hiroe, S. Noda, M. Nakauma, I. Asai, Mary K. Cowman, S. Al- Asaf, G. O. Phillips, 2007. Effects of the proteinaceous moiety on the emulsifying properties of sugar beet pectin. Food Hydrocolloids, 21, 1319-1329.

13. Relkin, P.,1998. Reversibility of heat-induced conformational changes and surface-exposed hydrophobic clusters of betalactoglobulin. Their role in heat-induced sol-gel state transition. Int. J. Biol. Macromol., 22, 59-66.

14. Sun S.F., 1994. Physical Chemistry of Macromolecules. John Wiley and sons Inc., USA.

15. Flory, P.J., 1953. Principles of Polymer Chemistry. Cornell University Press, New York.

16. Harding, S. E. 1997. The intrinsic viscosity of biological macromolecules. Progress in measurement, interpretation and application to structure in dilute solution. Progress Biophys. Mol. Biol., 68, 207-262.

17. Huggins, M. L., 1942. The viscosity of dilute solutions of long chain molecules. IV Dependence on Concentration $J$. Am. Chem. Soc., 64, 2716.

18. ZHOU jin-hua,WU-Yu xiong,SHEN Zhi-qiang. 2008. J. Cent. South Univ. Technol. 15, 520-524

19. Tanglertpaibul T, M.A. Rao, 1987. Intrinsic viscosity of tomato serum as affected by methods of determination and methods of processing concentrates. J. of food science, 52, 1642-1645.

20. Ibarz A, J. Pagan, R. Miguelsanz. 1992. Rheology of clarified fruit juices. II: Blackcurrant juices. Journal of Food Engineering, 15, 63-73.

21. Kraemer, E. O., 1938. Molecular weights of cellulose derivatives. Ind. Eng. Chem., 30, 1200.

22. Tanford, C., 1961. Physical Chemistry of Macromolecules, John Wiley and Sons Inc. USA.

23. Kar, F. and N. Arslan, 1999. Effect of temperature and concentration on viscosity of orange peel pectin solution and intrinsic viscosity-molecular weight relationship. Carbohydrate Polymers, 40, 277-284

24. Lapasin, R., and S. Pricl, 1995. Rheology of industrial polysaccharides: theory and application. Chapman and Hall, New York. 267-281

25. Rubinstein, M. and R. Colby, 2003. Polymer Physics, Oxford University Press: New York.

26. de Gennes, P. G., 1979. Scaling concepts in polymer physics. Cornell University Press, NY.

27. Morris G. A. and M. C Ralet, 2012. The effect of neutral sugar distribution on the dilute solution conformation of sugar beet pectin. Carbohydrate Polymers, 88, 1488-1491. 
